Information Engineering Express

International Institute of Applied Informatics

2016, Vol.2, No.4, $21-30$

\title{
A Pre-processing Approach for Fast and Stable Allocations on Approximation-based Pricing for Multi-unit Combina- torial Auctions
}

\author{
Naoki Fukuta *
}

\begin{abstract}
In this paper, some discussions about a pre-processing approach of fast approximation on stable pricing and allocation of resources in a combinatorial auction are presented. On the discussions, an approximate auction which has VCG-like pricing mechanism is used which considers the situation when a cancellation of winner bid(s) could be occurred after its completion of winner determination. An analysis about stable approximate pricing mechanisms against cancellation of a winner after its winner determination is also presented, where a single-unit non-combinatorial reserve price bidding on a combinatorial auction is employed on it. The pricing algorithms employ a kind of approximate allocation and pricing algorithms that are capable of handling multi-unit auctions with reserve price biddings. We consider a scenario on the allocation of electricity power usage rights while considering electricity generation costs on the power suppliers as well as external conditions such as violations of regulations done by some bidders outside the auction mechanism. An experimental analysis is presented on the scenario and the presented algorithms efficiently produced approximation allocations that are necessary in the pricing phase, while keeping the same level of stability in the case of single-winner cancellation scenario.
\end{abstract}

Keywords: combinatorial auction, approximation, algorithm

\section{Introduction}

Since it is crucial to realize dynamic allocations of limited resources with rational and self-interested attendees[17][1], numerous efforts have been done to provide better criterion and associated mechanisms to stably assign those limited resources, e.g., by fair allocations without money[2], or by efficient allocations using money[4].

In the context of realizing stable allocations using money, there have been proposed a number of variants of auctions to capture various conditions in the resource allocation problems[4]. For example, multi-unit auctions can handle amount

* Shizuoka University, Shizuoka, Japan 
of resources to be allocated for each bidder in an auction[22], and combinatorial auctions can handle complex allocations among different types of resources[23][4]. There exist numerous works on giving important theoretical properties, i.e., incentive compatibility[19][20], strategy proofness and other related properties[28], or considering locally envy free equilibrium[6] or similar properties[7] for more realistic scenarios. In these cases, stability of allocations is rather discussed in the context of how the allocations give the players incentives to follow the decisions made by the mechanisms while there could be some exceptions as discussed below.

There might be cases that one or more winners have been cancelled for allocating items that were auctioned[3], because of keeping revenue of the auctioneer, or just some winners did not have the rights to have them on a regulation. Such cancellations could be done by both buyers, sellers, and even by the auctioneers, due to some misconduct of the buyers or sellers. Although there are some ideas to prevent buyers from doing misconduct such as false name biddings, these mechanisms could not easily be realized when an auction would have other important characteristics[26]. Furthermore, such cancellations could be caused from the reasons that were completely outside of the mechanisms, e.g., a violation of low or regulations by the bidders' activities on the outside of the auction and its associated punishment has been applied to the bidders' rights that were also affected to the auction, even after the decision about the allocation has been made. Therefore, having considerations of such unexpected cancellations on an auction mechanism and its underlying algorithms is still an important issue to be discussed[10].

Because of the nature of combinatorial optimization problem, only a small change of its condition may affect to large parts of the allocation when we re-calculate its (sub)optimal allocations from scratch. In the above-mentioned context, we would say the allocation may not be stable for cancellations of bids. Although there is an approach to realize such a stability for a cancellations of bids[12], there are still some issues on its computation speed when it is applied to large-scale problems or massively repeated simulations.

In this paper, a preprocessing-based fast and stable approximate pricing algorithm against cancellation of a winner after its winner determination is presented ${ }^{1}$. In there, a single-unit non-combinatorial reserve price biddings on multi-unit combinatorial auction can also be employed to consider the costs of producing resources to be allocated. The approach should be helpful for numerous types of problems on making consensus among entities in difficult situations, e.g., some conflicts existed among those entities[18], as well as assigning limited amount of resources to self-interested entities (e.g., agents)[25].

\section{Preliminary}

\subsection{Multi-unit Combinatorial Auctions}

Combinatorial auction is an auction that allows bidders to place bids for a combination of items rather than a single item[4].

The winner determination problem on single unit combinatorial auctions is defined as follows[4]: The set of bidders is denoted by $N=1, \ldots, n$, and the set of

\footnotetext{
${ }^{1}$ Initial idea has been presented in [10], and further discussion has been done in [12].
} 
items by $M=\left\{m_{1}, \ldots, m_{k}\right\} .|M|=k$. Bundle $S$ is a set of items: $S \subseteq M$. We denote by $v_{i}(S)$, bidder $i$ 's valuation of the combinatorial bid for bundle $S$. An allocation of the items is described by variables $x_{i}(S) \in\{0,1\}$, where $x_{i}(S)=1$ if and only if bidder $i$ wins bundle $S$. An allocation, $x_{i}(S)$, is feasible if it allocates no item more than once, for all $j \in M$.

$$
\forall j \in M \quad \sum_{i \in N} \sum_{S \ni j} x_{i}(S) \leq 1
$$

The winner determination problem is the problem to maximize total revenue for feasible allocations $X \ni x_{i}(S)$.

$$
\max _{X} \sum_{i \in N} \sum_{S \subseteq M} v_{i}(S) x_{i}(S)
$$

When some items in auction can be replaceable each other, i.e., they are indistinguishable, the auction is called multi-unit auction. Multi-unit combinatorial auction is the case when some items are indistinguishable in a combinatorial auction[4]. Multi-unit combinatorial auction can be applied to electricity allocation problems, and other problems that considers quantitative or countable items in allocation problem[22].

In [13],[14], and [15], it has been shown that the presented hill-climbing approach outperforms SA[13], SAT-based algorithms[16], LP-based heuristic approximation approach[29], and a recent LP solver product in the setting when an auction has a massively large number of bids but the given time constraint is very hard.

\subsection{Winner Approximation and Pricing}

It is crucial for an auction mechanism to have a proper pricing mechanism to incentivize bidders to reveal their true valuations of items appropriately[4]. In VCG(Vickery-Clarke-Groves) mechanism[27][5], prices that winners will pay will be given as follows[24]. A payment $p_{n}$ for a winner $n$ is calculated by

$$
p_{n}=\alpha_{n}-\sum_{i \neq n, S \subseteq M} v_{i}(S) x_{i}(S)
$$

Here, the right part of the right side of the equation denotes the sum of all bidding prices of won bids, excluding the bids that are placed by the bidder $n$. The left part of the right side of the equation, $\alpha_{n}$ is defined by

$$
\alpha_{n}=\max \sum_{i \neq n, S \subseteq M} v_{i}(S) x_{i}(S)
$$

for a feasible allocation $X \ni x_{i}(S)$. This means that the $\alpha_{n}$ is the sum of all bidding prices of won bids when the allocation is determined as if a bidder $n$ does not place any bids for the auction.

In [24], Nisan et al. showed that optimal allocations should be used for VCGbased pricing to make the auction incentive compatible (i.e., revealing true valuations is the best strategy for each bidders). Also, Lehmann et al. showed that VCGbased pricing with approximate winner determination will not make the auction 
incentive compatible even when it is assumed that all bidders are single-minded(i.e., each bidder can only place single bid at each auction)[21].

To overcome this issue, Lehmann et al. prepared a special pricing mechanism that can only be applied for their approximate greedy winner determination[21]. However, this pricing mechanism can only be applied to their allocation algorithm but it cannot be applied to other approximation allocation algorithms. Also the mechanism is incentive compatible only when single-minded bidders are assumed[21].

The main problem in which VCG-based pricing is applied to approximation allocation of items is that there are the cases that: (1) the price for a won bid is rather higher than the bid price, and (2) the price for a won bid is less than zero, it means the bidder will win the items and also will obtain some money rather than paying for it[24]. In the situation of (1), it breaks individual rationality (i.e., the one will not pay a higher price than the placed bid when the one won the bundle of items). Also the situation of (2) is not preferable for both auctioneers and sellers.

To overcome the inability results shown in [24] and [21], a more relaxed condition called Strong Winner Price Monotonicity (SWPM) and an associated pricing algorithm transformToSWPM have been introduced[8], rather than applying the condition of strict incentive compatibility to a VCG-like pricing with approximation allocations.

As mentioned in [8], the algorithm transform ToSWPM is normally used with a good winner determination algorithm for the preparation of initial allocations to shorten the calculations for pricing. In the latter part of this paper, we assume that the $\mathrm{MHC}$ algorithm is used for providing initial allocations of items to those pricing algorithms, as described in [8].

\subsection{Approximate Pricing with Reserve Prices}

When approximately solving a combinatorial auction problem with reserve price biddings, a naive approach may produce a winner bid which includes a set of items whose prices are higher than the sum of reserve prices. For example, when we naively apply the pricing mechanism transform ToSWPM introduced in [8], the mechanism does not satisfy reserve price conditions(i.e., each winner places the price which is higher than the best combination of reserve price bids for the items in the bundle), since the mechanism used Lehmann's approximation allocation[21].

To overcome the issue shown in the previous part of this section, the algorithm transform ToSWPMRP that addresses the issue mentioned was presented[9][11].

\subsection{Approximation on Locally-strong Winner Price Monotonicity}

Here, we would consider a way to have a more relaxed condition than Strong Winner Price Monotonicity for better stabilization of pricing and re-allocation on bid cancellations. A weaker condition, Locally-strong Winner Price Monotonicity ${ }^{2}$, can be defined as follows:

Definition 1. (Locally-strong Winner Price Monotonicity: LWPM) For any non-empty bundle $s^{\prime} \subseteq S$ for a bidder $j \in N, X_{j}\left(s^{\prime}\right)=1$ (i.e., a winner) and whose bid price is $v_{j}\left(s^{\prime}\right)$, the Lehmann's greedy allocation of bids for the bundle of

\footnotetext{
${ }^{2}$ The idea has been initially presented in $[10]$
} 
items $s^{\prime}$ by bids from bidders $N^{\prime}=i \mid i \in N, i \neq j$ that satisfies $X_{i \neq j}\left(s^{\prime \prime}\right)=0$ (i.e., not winners) for any $s^{\prime \prime} \supseteq s^{\prime}$, always produces non-efficient allocation for the bundle $s^{\prime}$ (i.e., the total price of greedy allocation is less than $v_{j}\left(s^{\prime}\right)$ ).

Here, to satisfy this Locally-strong Winner Price Monotonicity, a modified version of the algorithm transform ToSWPMRP which is called transformToLWPMRP has been introduced[10], as follows:

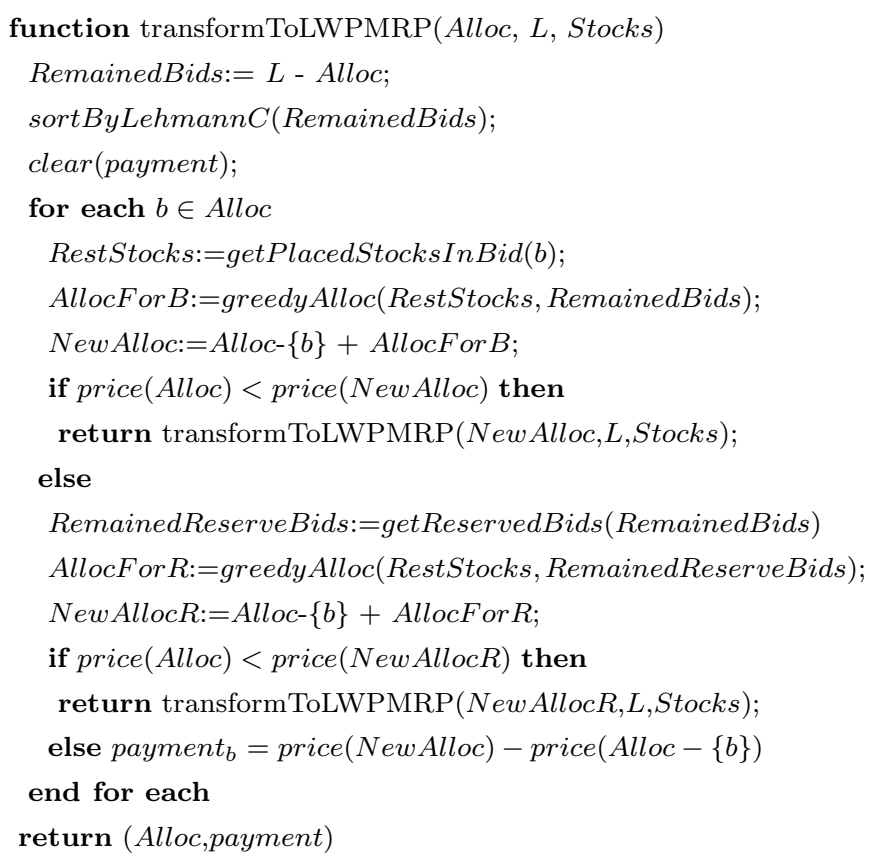

The algorithm transform ToLWPMRP applies its pricing and respective re-allocations of winners in a similar way that the algorithm transformToSWPMRP does. The major difference between them is which property is applied to justify the allocations and calculate the price to pay for each winner. On the algorithm transformToSWPMRP, it applied Strong Winner Price Monotonicity, while the algorithm transform ToLWPMRP applied Locally Strong Winner Price Monotonicity, instead, as both algorithms also applied Reserve Price Condition. For instance, the line 6 in the above algorithm is different. Here, a function getPlacedStocksInBid(b) returns the bundle of items that the bid $b$ placed to, as a set of pairs of the items and their unites to be won.

The two algorithms, transform ToLWPMRP and transformToSWPMRP were compared to see the differences on its allocation stability over pricing in the condition that any single winner has been cancelled on each auction. Here, the comparison has been done on how many winners have been removed from winners due to such single winner cancellation had been made and re-pricing had been applied. The auction problems that were used are the same datasets used in [11], one of which includes 1000 bidders and 100 percent of electricity production ratio, with 515024 bids and 67392 reserve-price bids, and another of which includes 3566 bidders and 100 percent of electricity production ratio, with 1710967 bids and 238584 reserve-price bids.

It is observed that the both algorithms produced the same number of winners for those auction problems. In [10], the comparison result in the case of 1000 bidders for 
the above scenario has been presented to analyze how those winners will be shifted when single winner has been cancelled and the respective pricing and allocation adjustment algorithms were applied. In this case, it was observed that the algorithm transform ToSWPMRP produced more losers that had been shifted from winners than that in the algorithm transform ToLWPMRP. On the algorithm transform ToLWPMRP, it produced approximately 87.5 percent $^{3}$ of such winner-shifts compared with transform ToSWPMRP[10]. Also a comparison in the case of 3566 bidders for the same scenario has also been done in the extended version of [10].

Although the algorithm transformToSWPMRP is tractable enough for a single problem and it produces its result much faster than that on ordinary VCG-based pricing mechanism, it still takes a long time when the scale of auction is huge. Furthermore, this makes it difficult to analyze how these kind of mechanisms make impacts to the behaviors of autonomous attendees in such auctions with a set of repeated simulations.

\section{Improving LWPM-checking Process of Stable Alloca- tion with Winner Cancellations}

\subsection{Faster Approximation on transformToSWPMRP}

To calculate Locally-strong Winner Price Monotonicity solutions faster, a modified version of the algorithm transform ToLWPMRP is proposed, which includes a mechanism to skip a part of its stability checking process in some conditions ${ }^{4}$.

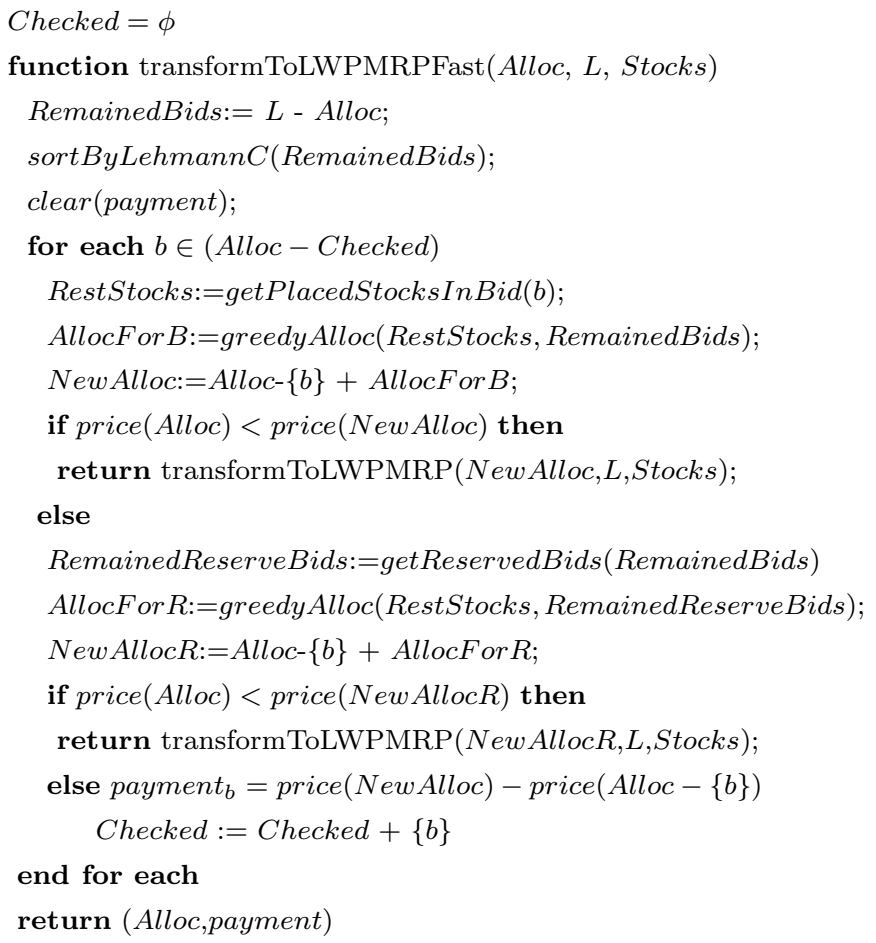

\footnotetext{
${ }^{3}$ This means it has been decreased from 857 to 750 .

${ }^{4}$ In [12], line 6 of the algorithm was accidentally wrongly printed without "- Checked" and it has been fixed in this version.
} 
The algorithm transformToLWPMRPFast efficiently omits the checking process of Locally-strong Winner Price Monotonicity once a winner has been checked in the algorithm. If the algorithm transformToLWPMRPFast is applied to a single-unit auction scenario, it guarantees Locally-strong Winner Price Monotonicity in case the order of getting $b$ in the line 6 is preserved as the order of allocation that has been done by greedyAlloc. A sketch of proof is that, in case a winner bid $b$ is said to be Locally-strong Winner Price Monotonicity satisfiable, there are no set of bids that can be replaced by the greedyAlloc function, since such bids should not remain in the remained bids nor they should not be in the Alloc since they should conflict to the winner $b$ when they are in the Alloc. However, in the case of multi-unit scenarios, the latter condition may not be guaranteed. Therefore, the algorithm transformToLWPMRPFast does not guarantee Locally-strong Winner Price Monotonicity by itself.

\subsection{Preprocessing Approach to Satisfy LWPM on transformToLW- PMRPFast}

Even when the algorithm transformToLWPMRPFast does not guarantee Locallystrong Winner Price Monotonicity, it can be used as a pre-processing to generate initial allocations for transformToLWPMRP. This will greatly improve the performance of transform ToLWPMRP. The improved algorithm transformToLWPMRPFastX can be prepared as follwos ${ }^{5}$ :

1: function transformToLWPMRPFastX(Alloc, L, Stocks)

2: (NewAlloc, $X):=$ transformToLWPMRPFast $($ Alloc, L, Stocks $)$

3: return transformToLWPMRP $($ NewAlloc, L, Stocks $)$

When this approach is applied to the case used in [10], the execution performance to complete its pricing process is improved. Table 1 shows how this transformToLWPMRPFastX can improve the computation performance while it also guarantees $L W P M$ as the transformToLWPMRP does. In the case of 3566 bidders, the whole computation with transform ToLWPMRPFastX to produce stable allocations has been done on approximately 523 seconds, while the computation with the pure transformToLWPMRP used in [10] could not complete its computation for this scale.

The proof of guaranteeing $L W P M$ on transform ToLWPMRPFastX is simply given by the fact that the transform ToLWPMRPFast in transform ToLWPMRPFastX is used as a pre-processing to generate an initial allocation for the computation in transformToLWPMRP and thus the all properties kept on it is also effective on transformToLWPMRPFastX.

Note that, in case the given initial solution (i.e., Alloc) is good enough and there is no chance to reallocate any winners in both transformToLWPMRPFast and transform ToLWPMRP, the computation time for transformToLWPMRPFastX will be roughly twice than that on just simply applying transformToLWPMRP. Since in that case the behaviors of transformToLWPMRPFast and transformToLWPMRP are identical, we can eliminate this overhead by just skipping transform ToLWPMRP in transform ToLWPMRPFastX and this can easily be implemented by adding a return value that indicates the number of reallocations done in the transformToLWPMRPFast.

\footnotetext{
${ }^{5}$ Note that the value in the variable $\mathrm{X}$ will not be used since the same value will be computed in transformToLWPMRP and returned from it.
} 
Table 1: Speed Improvement on Winner Cancellation Scenario with 1000 bidders

\begin{tabular}{r|r|r}
\hline & transformToLWPMRP & transformToLWPMRPFastX \\
\hline \hline Winners & 898 & 898 \\
Time of Computation (sec) & 1593 & 36.19 \\
\hline
\end{tabular}

\section{Conclusions}

In this paper, discussions about the preprocessing-based approach to realize faster computation of stable pricing and allocation of resources using an approximate auction which has VCG-like pricing mechanism when cancellation of winner bid(s) after its winner determination is considered.

As shown in the experimental result, the algorithm efficiently produced approximation allocations that are necessary in the pricing phase, while it keeps the same stability in the case of single-winner cancellation. As discussed in [11], it could behave as an approximation of VCG(Vickrey-Clarke-Groves) mechanism satisfying budget balance condition and bidders' individual rationality without enforcing the single-minded bidders assumption, while it does not guarantee strict strategyproofness.

In this paper, the presented analysis has been done only for a limited number of allocation scenarios for electricity usage in industries, although the mechanisms and the algorithms themselves could be applied to other problems such as bandwidth and channel allocation problem in wireless networks[23]. Applying to other possible scenarios and analyze how these pricing mechanisms affect the behaviors of attendees in an auction is one of our future work. Also further analysis on theoretical characteristics of the mechanism is future work.

\section{Acknowledgments}

The work was partly supported by Grants-in-Aid for Challenging Exploratory Research 26540162, Granst-in-Aid for Scientific Research(B) 15H02972, and JST CREST.

\section{References}

[1] B. An, V. Lesser, D. Irwin, and M. Zink. Automated negotiation with decommitment for dynamic resource allocation in cloud computing. In Proc. International Conference on Autonomous Agents and Multiagent Systems(AAMAS 2010), volume 1, pages 981-988, 2010.

[2] Ruggiero Cavallo. Incentive compatible two-tiered resource allocation without money. In Proc. the 13th International Conference on Autonomous Agents and Multiagent Systems (AAMAS2014), pages 1313-1320, 2014.

[3] Florin Constantin, Jon Feldman, S. Muthukrishnan, and Martin Pal. An online mechanism for ad slot reservations with cancellations. In Proc. of ACM-SIAM Symposium on Discrete Algorithms (SODA2009), pages 1265-1274, 2009. 
[4] Peter Cramton, Yoav Shoham, and Richard Steinberg. Combinatorial Auctions. The MIT Press, 2006.

[5] Sven de Vries and Rakesh V. Vohra. Combinatorial auctions: A survey. INFORMS Journal on Computing, 15(3):284-309, 2003.

[6] Benjamin Edelman, Michael Ostorvsky, and Michael Schwarz. Internet advertising and the generalized second price auction: Selling billions of dollars worth of keywords. American Economic Review, 97(1):242-259, 2007.

[7] Naoki Fukuta. A mobile agent approach for p2p-based semantic file retrieval. Journal of Information Processing, 20(3):607-613, 2012.

[8] Naoki Fukuta. An approach to VCG-like approximate allocation and pricing for large-scale multi-unit combinatorial auctions. Journal of Information Processing, 21(1):9-15, 2013.

[9] Naoki Fukuta. An approximation approach for large-scale multi-unit combinatorial auctions with reserve-price biddings. In Proc. The 2nd International Conference on Smart Computing and Artificial Intelligence(ICSCAI2014), pages 487-492, 2014.

[10] Naoki Fukuta. A preliminary analysis of allocation stability on approximationbased pricing for multi-unit combinatorial auctions - a single-winner cancellation scenario. In Proc. KICSS2015 International Workshop on Collective Intelligence and Crowd / Social Computing, pages 236-246, Phuket, Thailand, 2015.

[11] Naoki Fukuta. Toward efficient approximation for large-scale multi-unit combinatorial auctions with reserve-price bidding. IEICE Transactions on Information Systems, J98-D(6):948-961, Jun. 2015. (In Japanese.).

[12] Naoki Fukuta. Toward fast approximation of stable allocation and pricing on combinatorial auctions. In Proc. of 1st IEEE International Conference on Agents(ICA2016), pages 74-77, Sep. 2016.

[13] Naoki Fukuta and Takayuki Ito. Towards better approximation of winner determination for combinatorial auctions with large number of bids. In Proc. of The 2006 WIC/IEEE/ACM International Conference on Intelligent Agent Technology(IAT2006), pages 618-621, 2006.

[14] Naoki Fukuta and Takayuki Ito. Fine-grained efficient resource allocation using approximated combinatorial auctions-a parallel greedy winner approximation for large-scale problems. Web Intelligence and Agent Systems: An International Journal, 7(1):43-63, 2009.

[15] Naoki Fukuta and Takayuki Ito. An experimental analysis of biased parallel greedy approximation for combinatorial auctions. International Journal of Intelligent Information and Database Systems, 4(5):487-508, 2010.

[16] Holger H. Hoos and Craig Boutilier. Solving combinatorial auctions using stochastic local search. In Proc. of 17th National Conference on Artificial Intelligence (AAAI2000), pages 22-29, 2000. 
[17] Takafumi Ishikawa and Naoki Fukuta. A prototype system for federated cloudbased resource allocation by automated negotiations using strategy changes. In Proc. the Sixth International Workshop on Agent-based Complex Automated Negotiations (ACAN2013), 2013.

[18] Takayuki Ito, Yuma Imi, Takanori Ito, and Eizo Hideshima. COLLAGREE: A faciliator-mediated large-scale consensus support system. In Proc. of the International Conference on Collective Intelligence 2014, 2014.

[19] Takayuki Ito and David C. Parkes. Instantiating the contingent bids model of truthful interdependent value auctions. In Proc. of the International Joint Conference on Autonomous Agents and Multi Agent Systems (AAMAS2006), pages 1151-1158, 2006.

[20] Ron Lavi and Noam Nisan. Competitive analysis of incentive compatible on-line auctions. In Proc. of the 2nd ACM conference on Electronic commerce(EC2000), pages 233-241. ACM, 2000.

[21] Daniel Lehmann, Liadian Ita O'Callaghan, and Yoav Shoham. Truth revelation in rapid, approximately efficient combinatorial auctions. Journal of the ACM, 49:577-602, 2002.

[22] Kevin Leyton-Brown, Mark Pearson, and Yoav Shoham. Towards a universal test suite for combinatorial auction algorithms. In Proc. of ACM Conference on Electronic Commerce (EC2000), pages 66-76, 2000.

[23] John McMillan. Selling spectrum rights. The Journal of Economic Perspectives, 8:145-162, 1994.

[24] Noam Nisan and Amir Ronen. Computationally feasible VCG mechanisms. In Proc. of ACM Conference on Electronic Commerce, pages 242-252, 2000.

[25] Yoav Shoham and Kevin Leyton-Brown. Multiagent Systems: Algorithmic, Game-Theoretic, and Logical Foundations. Cambridge University Press, 2008.

[26] T. Todo, A. Iwasaki, M. Yokoo, and Y. Sakurai. Characterizing false-nameproof allocation rules in combinatorial auctions. In Proc. 8th International Conference on Autonomous Agents and Multiagent Systems (AAMAS2009), 2009 .

[27] William Vickrey. Counterspeculation, auctions, and competitive sealed tenders. The Journal of Finance, 16(1):8-37, 1961.

[28] Makoto Yokoo. The characterization of strategy/false-name proof combinatorial auction protocols: Price-oriented, rationing-free protocol. In Proc. of the 18th International Joint Conference on Artificial Intelligence, pages 733-739, 2003.

[29] Edo Zurel and Noam Nisan. An efficient approximate allocation algorithm for combinatorial auctions. In Proc. of the Third ACM Conference on Electronic Commerce (EC2001), pages 125-136, 2001. 